\title{
Estrategia de macrorreglas para mejorar la comprensión lectora
}

\author{
Macro-rules strategy to improve reading comprehension
}

Estratégia de macro-regra para melhorar a compreensão da leitura

\section{ARTÍCULO GENERAL}

César Loo Gil

cesarloo@biofab.com.pe

https://orcid.org/0000-0001-8396-5972

CientificoInvestigador de BioFab Inc.y del Centro de Investigación \& Producción Científica IDEOs, Miraflores - Perú
Edward Rodriguez Mendoza edward.rodriguez1@unmsm.edu.pe https://orcid.org/0000-0001-8052-9614 Universidad Nacional Mayor de San Marcos, Lima - Perú
Tania De La Cruz Barriga taniadelacruzb@gmail.com https://orcid.org/0000-0001-8169-4195 Especialista en Educación de la UGEL Norte de Arequipa, Perú

Recibido 21 de Octubre 2021 | Arbitrado y aceptado 9 de Enero 2022 | Publicado en 28 Abril 2022

\section{RESUMEN}

La motivación de este artículo se origina en la realidad de la educación en el Perú, la cual se encuentra por debajo de lo optimo más del $50 \%$ de los alumnos de segundo y cuarto año de primaria no saben leer correctamente-, por otro lado, la forma en que se enseña a comprender a leer no es correcta. En base a esta desalentadora realidad, este artículo presenta estrategias para amenizar el proceso y la experiencia del aprendizaje, como las estrategias comunicacionales para practicar la lectura; el fomento de la escritura, para ayudar al proceso de retroalimentación; o las actividades lúdicas, para hacer divertido el aprendizaje. Además, también se presentan a las cuatro macrorreglas: omisión, construcción, generalización, e integración, como herramientas para comprender mejor lo que se lee.

$$
\begin{aligned}
& \text { Palabras clave: } \\
& \text { Estrategias de } \\
& \text { comprensión lectora }
\end{aligned}
$$

\section{ABSTRACT}

The motivation for this article originates from the reality of education in Peru, which is below optimal -more than $50 \%$ of second and fourth year primary school students cannot read correctly-, on the other hand, the way in which reading is taught to understand is not correct. Based on this discouraging reality, this article presents strategies to liven up the learning process and experience, such as communication strategies to practice reading; the encouragement of writing, to aid the feedback process; or playful activities, to make learning fun. In addition, the four macro rules: omission, construction, generalization, and integration are also presented as tools to better understand what is read.

Key words: Macro rules; Teaching strategies; reading comprehension

\section{RESUMO}

A motivação para este artigo origina-se da realidade da educação no Peru, que está abaixo do ideal - mais de $50 \%$ dos alunos do segundo e quarto ano do ensino fundamental não sabem ler corretamente -, por outro lado, a forma como se ensina a ler não está correto. A partir dessa realidade desanimadora, este artigo apresenta estratégias para dinamizar o processo de aprendizagem e a vivência, como estratégias de comunicação para a prática da leitura; o incentivo à escrita, para auxiliar no processo de feedback; ou atividades lúdicas, para tornar $\mathrm{o}$ aprendizado divertido. Além disso, as quatro macro regras: omissão, construção, generalização e integração também são apresentadas como ferramentas para melhor compreender o que é lido.

Palavras-chave: Macro regras; Estratégias de ensino; compreensão de leitura 
Introducción

La educación en el país se encuentra por debajo de los estándares internacionales, existen problemas de contenido y de metodología, que no nos permite educar correctamente a nuestros niños. En lo concerniente a la enseñanza de la comprensión lectora, los resultados de las pruebas que el gobierno peruano aplica a los estudiantes, son desalentadores, porque nos demuestra que, para la segunda década del siglo XX, aún no sabemos leer correctamente.

Los resultados de la última Evaluación Censal de Estudiantes (ECE), aplicada en el 2016 por el Ministerio de Educación, no fueron nada alentadores: solo el 46,4\% de los estudiantes de segundo grado de primaria alcanzaron un nivel satisfactorio en lectura. En cuarto de primaria, incluso, la cifra fue aún más baja: $31.4 \%$. Según el Instituto de Estadística e Informática, en ese mismo año, 8 millones 668 mil alumnos fueron matriculados en el Sistema Educativo Nacional. Es decir, casi 4 millones de estudiantes no comprendía lo que leía. (El Comercio, Lima, 6 de agosto 2018)

La última prueba de la Evaluación Censal de Estudiantes (ECE), aplicada a 165658 alumnos de segundo y cuarto de secundaria, de 5976 escuelas sobre matemáticas y comprensión lectora ha dado los siguientes resultados: El $62.4 \%$ de los alumnos de segundo grado de primaria, que rindieron la prueba se encuentran en inicio y en proceso de aprendizaje y solo el $37.6 \%$ se encuentra en el nivel satisfactorio. A diferencia de los resultados obtenidos en 2018 (37.8 \% satisfactorio) y del 2016 (46.4\% de satisfactorio), lo que evidencia que se ha retrocedido en la calidad de la enseñanza o si se prefiere la sutileza, se ha desaprendido. Por otro lado, los resultados de los alumnos de cuarto grado de primaria son aún más desalentadores, $65.5 \%$ se encuentra en los niveles previo al inicio, en inicio y en proceso, solo el 34.5\% se encuentra en el nivel satisfactorio.

(MINEDU, 2019)

La lectura como un conjunto de habilidades y transferencia de conocimiento es un enfoque nacido en los sesenta, que define aprender a leer como el reconocimiento de grafías, comprensión del contenido y finalmente la evaluación del contenido. Hacer que aprender a leer, y a comprender lo que se lee debe ser una experiencia amena para el educando, por lo que los maestros deben entender que lo que enseñar es una transferencia entre alumno y maestro, y se deben asumir estrategias que permitan una transacción armoniosa.

La lectura entendida como un proceso y como una experiencia de aprendizaje, como un trabajo de construcción de significados que se realiza mediante las transacciones que se 
establecen entre el lector, el texto y el contexto (Rosenblatt, 1994). Las intervenciones docentes que integran este conjunto dan cuenta del reconocimiento de este proceso de lectura en el aula, y se orientan a la habilitación de múltiples interpretaciones, la validación de las mismas y las síntesis que permiten dar cierres parciales para avanzar a lo largo de las actividades previstas. (Ripoll, 2016, p. 43)

Este enfoque permite conseguir una relación más amena del lector con el texto, podemos decir un dialogo entre autor y lector. Aprender a leer tiene dos componentes, el primero subjetivo, es encontrar la motivación adecuada para el educando, haciendo que leer sea placentero; el segundo componente es objetivo, y este tiene que ver con herramientas y enfoques metodológicos para alcanzar estándares óptimos de comprensión lectora.

Comprender a leer es una de las habilidades más importantes, en el proceso de maduración cognitiva del ser humano; así también, es de suma utilidad para la vida diaria, como especifican diversos autores (Sepúlveda y Martínez, 2018; Hyman y Díaz, 2018; Rodríguez, 2019), comprender a leer requiere de esfuerzos y métodos que acerquen la educando a una experiencia amena de forma transaccional entre autor y lector.

Visto el problema que afecta a la educación en el Perú, así como, los enfoques para enseñar a leer, podemos revisar algunas estrategias para su enseñanza. Existen pues disparidades en la enseñanza (Grade, 2017), que se originan en diversos tópicos, como demanda, recursos, matrículas, entre otros.

Frente a este problema, creemos que aplicar una metodología basada en la perspectiva del niño, además de enseñar contenidos amenos, va facilitar la enseñanza del educando. De este modo, creemos que la enseñanza de las a través de estrategias de enseñanza como las comunicacionales, el fomento de la escritura, las actividades lúdicas y las macrorreglas van ayudar a los niños a comprender lo que leen, puesto que, se va generar un apego, a través de la experiencia amena; a su ves que se utilizan herramientas que permiten que el lector filtre la información y aprehenda lo esencial del texto.

Este artículo busca, exponer las ventajas de usar las estrategias para construir una experiencia amena en los educandos, así como, brindarles herramientas para que puedan comprender de manera correcta lo que están leyendo. Se muestran estrategias que buscan lograr un vinculo con la lectura, para que no sea aprenderla algo tedioso y aburrido, sino por el contrario ameno y divertido.

Dicho de otra forma, "La comprensión lectora hace referencia a un proceso simultaneo de extracción y construcción transaccional entre las experiencias y conocimientos del lector, con el texto" (Gutierrez-Braojos y Salmerón, 2012) 
Este artículo se organiza en las bases metodológicas, las estrategias para enseñar a leer, y la definición y explicación estratégica de las macrorreglas. Y para concluir, se expresan la crítica, el análisis y las conclusiones.

\section{Metodología}

La metodología empleada para la realización de este artículo se basa en cinco puntos:

- $\quad$ Búsqueda bibliográfica

- $\quad$ Criterios de selección

- $\quad$ Evaluación de la calidad de la bibliografía seleccionada

- $\quad$ Uso de documentos oficiales

- $\quad$ Análisis de la viabilidad, fiabilidad y valides de la bibliografía.

Estrategias para enseñar a leer

En señar a leer, es un proceso dinámico, pues se basa en la relación educador-educando. En este proceso, el educador instruye al alumno a poder reconocer y comprender grafías y el educando responde a la instrucción con aciertos y desaciertos, propios del proceso educativo; sin embargo, lo que se espera es que al finalizar el proceso los alumnos puedan leer, y sobre todo, comprender lo que leen. Pues bien, no siempre ocurre lo esperado, cabe decir que en nuestro país ocurriría lo contrario en gran medida. Enseñar a los niños es más sencillo, si lo que se enseña es de su agrado; hacer que el niño tenga interés es primordial, pues al obtener a atención del educando lo que se pretende enseñar cala con mayor facilidad en él. Facilitar la comprensión implica que el docente intervenga de manera activa en el proceso, ayudando a construir una experiencia amena, Bustos et al (2017), menciona que intervenir en dicho proceso reduce el tiempo y esfuerzo para cumplir con la meta.

Estas interacciones pedagógicas buscan acortar la distancia entre un texto y un lector que aún no está preparado para representarse su significado autónomamente dado el número de habilidades que debe poner en juego y cuyo tiempo de desarrollo, a nivel experto, podría demandar un tiempo importante de la formación escolar (ver estudio de Ericcson, 2002 sobre el tiempo de práctica en tareas de alta complejidad). (Bustos et al, 2017, p. 91)

Por ello, consideramos que utilizar estrategias para conseguir la atención del estudiante, y facilitar su aprendizaje es prioritario, y por ello a continuación se explicaran algunas propuestas.

Estrategias comunicacionales 
Las estrategias comunicacionales hacen referencias a la búsqueda continua de la lectura, esto quiere decir que los maestros deben buscar que los niños lean y hablen todo el tiempo posible, al interior y exterior de las escuelas.

Esto se puede lograr a través de adivinanzas, fichas, imágenes, canciones, etc. Que generen en los educandos la necesidad de leer y comentar lo que han leído.

Las adivinanzas son juegos de palabras en lo que se alude algo que no está explícito. En algunas se ofrece una descripción y en otras se utilizan metáforas de lo que hay que adivinar. Promueven el análisis y la deducción. (León, 2017, p. 30)

Otro ejemplo podría ser pegar notas en el refrigerador o en la puerta de la habitación del niño; practicar la lectura con pequeñas dinámicas como leer los anuncios en las calles. De esta forma, los niños van adquiriendo el habito de leer, comentar y expresar lo que sienten o creen al respecto.

[...] Específica que el educador debe propiciar espacios de expresión oral utilizar diversas estrategias como los juegos verbales o lingüísticos, que permite que el niño desarrolle la expresión y comprensión oral, que incremente su vocabulario, que discrimine los sonidos de las palabras, que ejercite de manera lúdica los músculos de la boca, para conseguir una adecuada pronunciación y fluidez en el habla. (León, 2017, p. 29)

La escuela se basa, principalmente, en enseñar a través de un texto único de lecturas; esto puede ser útil para marcar las pautas de los temas a tocar, pero deben ser unidades de enseñanza cortas que paulatinamente se vayan alargando, con el incremento de la capacidad de comprensión del educando. Por otro lado, no es solo la extensión del texto, de que debe ser variado, sino también los temas. El enfoque tradicional señala lecturas que versan sobre un contenido determinado, ciencia, literatura, noticias, entre otros; pero no se ha considerado los intereses y la cotidianidad de los educandos, esto quiere decir que el texto educativo debe ser ameno, divertido-placentero- así como, debe generar un recuerdo o evocar familiaridad, para lograr que el educando pueda entenderlo con mas facilidad y pueda expresarse sobre el tema.

En las escuelas de educación básica regular, se enseña en base a textos con contenidos detallados e inmodificables. Estos textos, versan sobre contenidos que muchas veces son ajenos a los niños, versan sobre ciencias y literatura, tópicos que quizá el alumno no ha visto y no comprende. Hay un desperdicio de tiempo y energía en explicar aquello que el educando desconoce. Por otro lado, si el texto fuese una guía temática, y la elección de los textos se basaran en aspectos que el alumno conoce, temas cotidianos o de su agrado, 
se lograría ahorraría estos recursos, además de generar interés y lograr una experiencia amena.

Fomento de la escritura

Otra estrategia de escritura es el fomento de la escritura, debido a que al escribir se relacionan significantes con significado, es decir la palabra (o letra) con una representación. Por lo tanto, las lecturas cortas, amenas y familiares van a propiciar que el alumno escriba con facilidad, y así retroalimente el proceso cognitivo de leer. De esta manera, la escritura de la palabra casa estará asociada a la imagen de una casa, haciendo que leer sea mas comprensible. La enseñanza de la lectoescritura es enseñada en las escuelas a través de la escala de complejidad-fácil difícil-sin embargo, los niños asocian los significados desde lo general a lo especifico.

Escribir: A la destreza de leer le corresponde la destreza de escribir, esto fortalece la comunicación, al escribir se deja el menaje perdurable en el tiempo, pues la escritura ha permitido el desarrollo del conocimiento en la sociedad. (Arizaga, 2020, p. 19)

Actividades lúdicas

Enseñar a leer mediante actividades que entretengan, no solo en los primeros años de educación, sino a lo largo de todo el periodo básico de enseñanza. Enseñar desde esta perspectiva puede ayudar a que el educando no considere aburrida o tediosa la lectura.

Para la enseñanza en primaria, se pueden enseñar a través de imágenes, de trabalenguas, adivinanzas, entre otros; así también a escribir con diferentes tipos de letras -fuentes en el lenguaje computacional- además de usar colores. La mayoría de veces los profesores indican al educando a escribir monocromáticamente, sin embargo, usar colores puede fomentar su creatividad, hacer la escritura amena, y así entender y atribuir significados propios con más facilidad.

Las actividades lúdicas, como parte de la didáctica, han sido utilizadas en la educación para la resolución de problemas de diverso índole y en las distintas áreas del conocimiento humano; ya que el juego, que es el centro de las actividades lúdicas, es una actividad que genera un estado de bienestar y alegría, que impulsa el desarrollo integral de la personalidad y la capacidad creadora de la persona. (Arizaga, 2020, p. 20)

Por ejemplo, crear dinámicas favorece al proceso de aprendizaje, porque obliga al niño a buscar en su repertorio de palabras, es decir, buscan la palabra-escrita y dicha- que cumpla con los requerimientos de la dinámica. Una dinámica podría ser la siguiente, decir una palabra, fuego, describirla y contextualizarla, con la finalidad de que comprenda los diferentes valores de dicha palabra. El fuego, es de color rojo o anaranjado, es muy 
caliente, sirve para cocinar o calentar la comida; mucho fuego puede ser peligroso, mucho fuego puede ser un incendio.

El uso de las palabras constituye un juego en sí, pues existe analogías en ellas, ellas connotan diferentes significados. Por ejemplo, en el lenguaje oral pollo y poyo, son palabras homófonas, que aluden a un ave y aun asiento de piedra; por otro lado, cepillo, puede aludir a higiene dental, carpintería, peinado, entre otras actividades, y aun en otros sentidos una palabra puede tener un significado para un contexto determinado, por ejemplo, para Luis la palabra pollo significa ave comestible, así como también, su mejor amigo, pues Enrique es conocido como pollo. Guerra (2019) menciona que el lenguaje en si es una actividad lúdica, pues en base a lo que Wittgenstein (1976), denomina juegos del lenguaje.

[...] múltiples ejemplos de juegos, como hacer preguntas y responderlas, dar una orden, describir un objeto, hacer un reporte de un evento, inventar historias, resolver problemas aritméticos, y un largo etcétera. (Guerra, 2019, p. 64)

Para Wittgenstein (1976), las palabras dependen del contexto y la intencionalidad, porque ello genera dimensiones análogas.

[...]la existencia de las palabras "pensar" y "pensamiento" junto a las palabras que denotan actividades (corporales), tales como escribir, hablar, etc., nos hace buscar una actividad, diferente de éstas, pero análoga a ellas, que corresponda a la palabra "pensar". Cuando las palabras tienen prima facie en nuestro lenguaje ordinario gramáticas análogas, nos inclinamos a intentar interpretarlas análogamente; es decir,tratamos de hacer valer la analogía en todos los campos. (Wittgenstein, 1976, p 36)

Por lo tanto, la dinámica o el juego, potencian el aprendizaje. Rosembreg (2018), menciona que el lenguaje y el juego se relacionan bidireccionalmente, y se potencian entre sí; además, el lenguaje es el soporte del juego, debido a ser el principal instrumento de la actividad, el lenguaje se entrelaza con las acciones para crear el objetivo del juego. El lenguaje y el juego tienen una relación bidireccional, una relación de influencia mutua, se potencian entre sí. Por una parte, el lenguaje, en su carácter de principal instrumento de pensamiento para categorizar experiencias (Nelson, 1996) se presta, por medio de la comunicación selectiva de significados, a la representación y recreación de estas experiencias en el marco del juego. El lenguaje se entrelaza con la acciones de los participantes y contribuye a crear la estructura y la trama del juego. Permite que el juego se despliegue y se sostenga en el tiempo. Por otra parte, al jugar, se aclaran los componentes representacionales que subyacen al juego y se promueven distintos aspectos 
del desarrollo lingüístico y discursivo de los niños. De este modo, el juego se torna una situación de enseñanza informal en la que se aprende el lenguaje. (Rosemberg, 2018, p. 1)

De esta manera, se ha tratado de expresar que en nuestro país existe un problema aun sin solucionar, que es la enseñanza de la comprensión lectora. Las evaluaciones que realiza el estado, muestran una realidad que no puede esconderse ni ocultarse debajo de la alfombra. Por ese motivo, aplicar en la enseñanza diferentes estrategias que abarquen, la forma de enseñanza, asi como el contenido de la enseñanza con la finalidad de hacer mas entendible y practica la lectura.

Las macrorreglas para la comprensión lectora Definición

Las macrorreglas son herramientas que se utilizan en la comprensión de textos expositivos, con la finalidad de conseguir un desagregado de información que se conecta formando el binomio micro tema y macro tema, estas conexiones proposicionales nos permiten comprender lo que el autor no quiere decir, eliminado información innecesario o repetitiva. Diversos autores (Palomino, 2017; Londoño y Ospina, 2017; Roncales, 2015; Vela, 2017; Serrano, 2016; Villalobos y Uceda, 2020), señalan la importancia de las macrorreglas de Van Dijk, en el proceso de la comprensión lectora, de manera indistinta a los grados de instrucción, es decir que pueden y deben ser usadas por niños de primaria y por adultos universitarios. Pues estas macrorreglas permiten la transacción de información entre el autor y el lector.

Las macrorreglas son un conjunto de reglas propuestas por el sociólogo y lingüista neerlandés Teun Van Dijk (1978), que nos permiten entender la unión de las proposiciones y la relación entre los diferentes niveles semánticos. Las macrorreglas nos permiten crear ideas a partir de ideas más pequeñas.

Así pues, necesitamos reglas para la realización de la unión de micro- y macroestructuras, que se evidencian como series de proposiciones ligadas a series de proposiciones, puesto que en ambos casos se trata de estructuras significativas proposicionales. (Van Dijk, 1978, p. 58)

Las macrorreglas según Van Dijk (1978) son cuatro: Omitir, Seleccionar, Generalizar, e Integrar. Estas macrorreglas deben estar cumplir con el principio de implicación semántica, que indica que cada producto de las macrorreglas debe pertenecer o estar implicada en una anterior.

Las reglas de Van Dijk, surgen con la finalidad de estudiar las estructuras detrás del discurso, en un enfoque sociológico; cuya utilidad no solo es útil en sociología, sino 
también en la educación y la pedagoía. (Mejía, Cortés y Velásques, 2016; Acaro, 2019; Pallares y Rodríguez, 2016)

Para comprender mejor a lo que se refiere Van Dijk (1978), veamos el siguiente ejemplo:

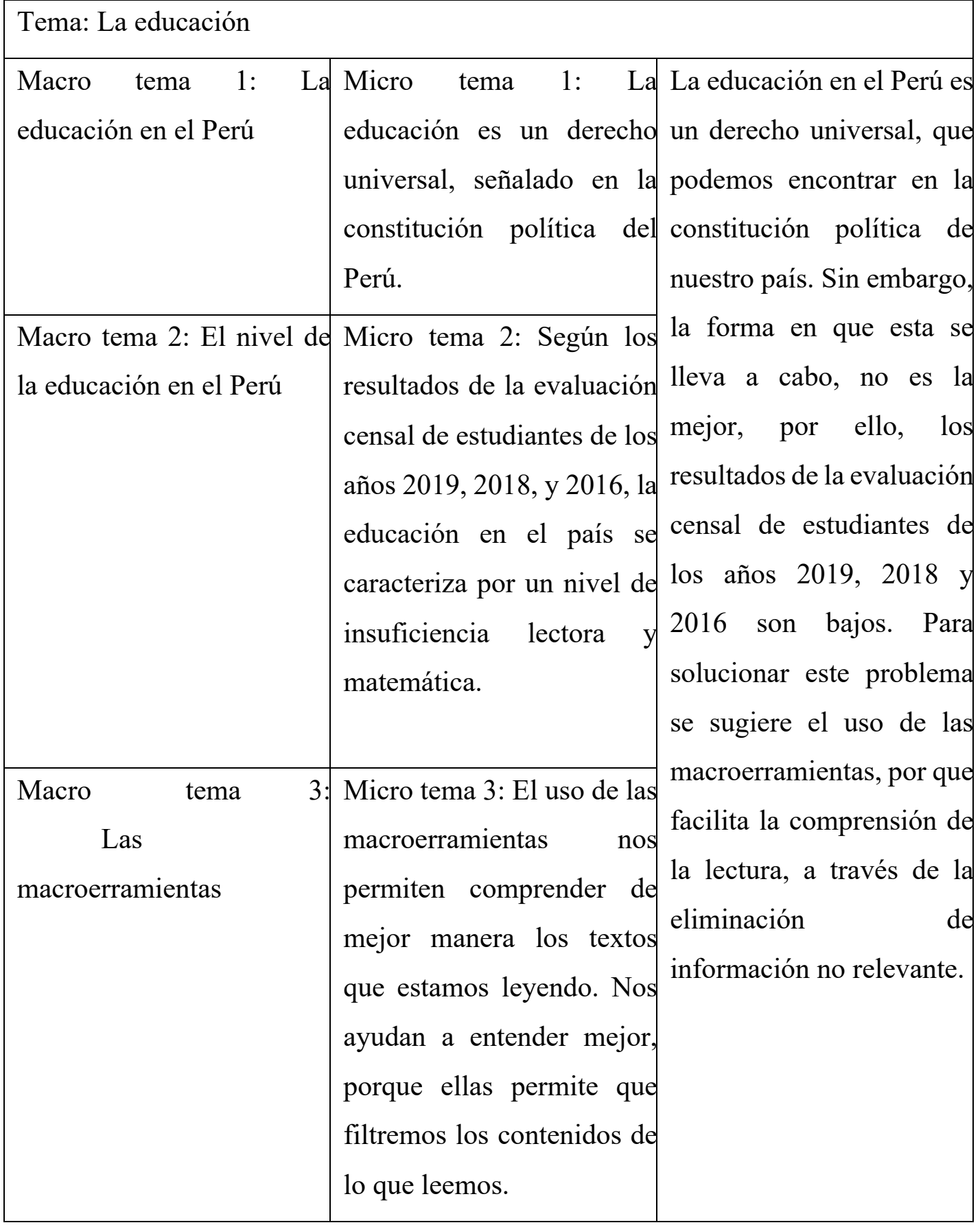

En base al ejemplo anterior podemos apreciar cómo vamos desagregando el contenido de la información, en micro temas y macro temas. Como se puede leer en el ejemplo anterior, 
las ideas del texto pueden ser clasificadas en diversos subtemas, como La educación en el Perú, y lo que puede ser considerado el contenido del subtema La educación es un derecho universal, señalado en la constitución política del Perú. Dentro de un tema que lo engloba: La educación.

Las macrorreglas se enfocan en el entendimiento de los textos, análisis del discurso, con la finalidad de entender cual es la perspectiva del autor, respecto al tema desarrollado. Julcamayan (2019), describe el uso de las macrorreglas dentro de la lingüística textual, que es aquella lingüística que permite el vinculo de distintas disciplinas. Esto quiere decir que las neurociencias, la psicología, la sociología, la educación o las matemáticas pueden dialogar gracias a la lingüística del texto.

Las macrorreglas se enmarcan en la lingüística textual o análisis del discurso, ya que se aplican en textos. Estos textos son propicios para el establecimiento de macrorreglas o macroestrategias dado carácter comunicativo y composición en proposiciones que dan cuenta de la macroestructura. La lingüística textual permite relacionar la lingüística con otras disciplinas, pues tiene carácter interdisciplinario. De esta manera también se tiene como fundamento la psicología cognitiva debido a que la coherencia y la interpretación son difícilmente asimilables si no existe la comprensión lingüística y gramatical de la estructura del texto (Van Dijk, 1978). (Julcamayan, 2019, pp. 72-73)

a) Omisión: Esta regla permite al lector suprimir todas las ideas que puedan ser irrelevantes o redundantes, por lo que se pueden suprimir porque no contribuyen a entender el resto del texto. Por ejemplo, en el mundo de las aves, las águilas son uno de los mayores depredadores, estas aves suelen comer a otras aves como palomas, patos, garzas y otras aves más pequeñas. En este ejemplo podemos eliminar patos y garzas, porque son redundantes para comprender la oración.

b) Construcción: Esta regla se encarga de organizar las ideas de texto en macro temas, e integrar ideas micro para cada tema macro, así también, suprime ideas irrelevantes o redundantes.

c) Generalizar: Esta regla indica que las ideas de un texto pueden ser clasificadas en criterio general. Por ejemplo, perro, vaca, conejo pueden ser clasificados en mamíferos

d) Integración: Esta regla indica que las ideas del texto pueden ser integradas en una sola idea, que las represente. Por ejemplo, perro, vaca, conejo, pueden ser reemplazados por perro, y relacionarse con mamíferos.

Como explican Briceño et al (2019) sobre las macrorreglas, "son una reconstrucción de aquella parte de nuestra capacidad lingüística con la que enlazamos significados 
convirtiéndolas en totalidades significativas más grandes”. (Briceño et al, 2019, p. 31)

La estrategia de la macrorreglas

Las macrorreglas, como se explicado en líneas anteriores, son preceptos que no permite dilucidar el contenido de una oración, con la finalidad de entender las relaciones semánticas que hay dentro de ella. Las macrorreglas son cuatro: Omitir, Seleccionar, Generalizar, e Integrar. La mayoría de textos que encontramos en el periodo de educación básica, son de tipo expositivo. Este tipo de texto se caracteriza por explicar un determinado tema desde la perspectiva del autor. Sobre este tipo de texto aplicaremos las macrorreglas.

Podemos definir, que la estructura de un texto esta conformada por varias proposiciones que se enlazan por conexiones semánticas, que pueden ser explicitas o tacitas. La macrorreglas se engranan en dos niveles: Microestructura y macroestructura.

Microestructura: Esta estructura hace referencia al contenido descriptivo de un texto, esta estructura enriquece al texto, pues se conforma de epítetos y generan la particularización del tópico.

Por ejemplo: los perro, lobos, coyotes y chacales son coespecies del genero canis. Por otro lado, los gatos, los linces, leones y tigres pertenecen a la familia felinae. Todos estos animales son carnívoros.

Macroestructura: Esta estructura versa de manera generalizada sobre un tema, estableciendo los parámetros o limites del texto.

Por ejemplo: caninos y felinos.

Ambas estructuras se van generando en simultaneo, ya que cuando se va aprehendiendo la microestructura, se va desarrollando la macroestructura de esta forma, al entender que los perros, lobos, coyotes y chacales son coespecies del genero canis. Por otro lado, los gatos, los linces, leones y tigres pertenecen a la familia felinae. Todos estos animales son carnívoros, nos estamos refiriendo a caninos y felinos, la macroestructura. Dilucidar la microestructura es descubrir la macroestructura.

La comprensión del texto requiere de comprender dos aspectos, el primero es el textual, en el cual el lector entiende el contexto sociocultural del texto-comprender al autor-. Lo que refiere a que entender, características particulares e inherentes a un determinado texto.

Para poder facilitar la comprensión del texto, y así poder usar las macrorreglas con mayor facilidad y comprensión, es necesario tener en cuenta que hay dos tipos de comprensiones: Literal e Inferencial. 
A. Comprensión literal: Este tipo de comprensión se basa en aprehender elemento o ideas del texto, tales como características, localizaciones, relaciones semánticas, temas principales y secundarios, entre otros. La utilidad de este tipo de comprensión recae en lograr que el lector recuerde, reconozca y relacione las ideas. El nivel de comprensión literal se basa en reconocer las ideas explícitas.

B. Comprensión inferencias: Este tipo de comprensión indica al lector que existe un nivel implícito, y muchas veces no textual, donde las ideas deben ser aprehendidas por deducciones y procesos de análisis. Conjeturas, hipótesis, inferencias, son informaciones que el lector debe descubrir. En este tipo de comprensión, por ejemplo, encontramos características ocultas de un personaje, características o posiciones del autor, juicios de valor, la subjetividad del creador del texto.

Por lo tanto, las macrorreglas nos ayudan a poder desarticular un texto complejo, para poder entender las ideas que se encuentran en su interior. Como se ha visto en líneas anteriores, fomentar el gusto por la lectura en los educandos, ya que el gusto por la lectura facilita la comprensión de los textos debido a la predisposición del niño. Usar colores, actividades lúdicas, estrategias comunicacionales y fomentar la escritura logran generan el interés en los educandos. Por otro lado, la estrategia de las macrorreglas complementan a las demás estrategias para lograr una comprensión de lectura que abarca las dimensiones subjetiva y objetiva, al brindar la herramientas para comprender, desde un enfoque adecuado y logrando afinidad por la lectura en los educandos.

Critica

La principal crítica que se puede ser al respecto de lo expuesto es sobre la metodología y las motivaciones de la enseñanza. Existe pues una desmotivación de los maestros, explicada por diversos argumentos: bajos salarios, exceso de carga lectiva, entre otras. Lo cual repercute en la calidad de la enseñanza, así como la que recibe el educando. Problemas estructurares del sector educación. En base a lo expuesto, creemos que hacer amena, divertida y familiar la lectura, motivará al estudiante a aprender, y usar las macrorreglas, ayudará al proceso de aprendizaje, con mayor efectividad, pues el alumno estará predispuesto y motivado.

Análisis y conclusiones

En base a lo expuesto en este artículo, podemos afirmar que la educación en el país se encuentra en un periodo crítico, ya que no estamos a la par de otros países de la región y 
muy por debajo de países del llamado primer mundo. Es en base a ello, creemos que conseguir la atención y que el alumno se sienta interesado por la lectura es de suma importancia, ya que esta manera no sentirá que el proceso y la experiencia de leer es tediosa y aburrida, por el contrario, le será amena. De esta manera, la transacción de información y subjetividades entre el lector y el autor será cordial; ahora, por otro lado, usar las macrorreglas: Omisión, Construcción, Integración, y Generalización, ayudaran a comprender de mejor manera lo expuesto por el autor, a través de la supresión de información irrelevante o redundante, y así pueda obtener lo esencial del texto.

Bibliografía

Acaro, A. (2019). Propuesta de estrategias didácticas, basada en la teoría de van dijk para mejorar los niveles de comprensión lectora de los estudiantes del tercer grado de primaria, i. e. "carlos a. salaverry"- la victoria. chiclayo, 2018,(Tesis de maestría), Universidad Nacional Pedro Ruiz Gallo, Perú.

Arizaga, A. (2020). Estrategias metodológicas lúdicas para reforzar las macro destrezas leer y escribir en el Área de Lengua y Literatura. Una propuesta para aula de apoyo "Santa Clara de Asís" de la Posada San Francisco. (Tesis de licenciatura). Universidad del Azuay, Ecuador.

Briceño et al (2019). Aplicación de las macrorreglas de Van Dijk para mejorar la producción de textos expositivos-I.E. República de Panamá N81001 de Trujillo, 2018. (Tesis de licenciatura). Universidad Nacional de Trujillo, Perú.

Bustos et al (2017). Leer para aprender: Cómo interactúan los profesores con

El Comercio (2020). Comprensión lectora: una tarea pendiente en el Perú. [Revisado el 30 de noviembre del 2020] Recuperado de: https://elcomercio.pe/peru/comprensionlectora-tarea-pendiente-peru-videonoticia543914-noticia/?ref=ecr

Grade (2017). Estado de la educación en el Perú Análisis y perspectivas de la educación básica. Impresiones y Ediciones Arteta E.I.R.L., Perú

Guevara, J. (2019). Desarticulando la evolución del lenguaje. Wittgenstein y los juegos de lenguaje con animales. Tabula Rasa, 32, p. 57-80

Gutiérrez-Braojos, C. y Salmerón, H. (2012). Estrategias de comprensión lectora: enseñanza y evaluación en educación primaria. Profesorado, 16(1), p. 183-202. 
Hyman, D. y Díaz, T. (2018). Orientaciones didácticas para el fortalecimiento de la comprensión lectora. (Tesis de maestría). Universidad de la costa, Colombia.

Julcamayan, C. (2019). El uso de las macrorreglas en la comprensión de textos expositivos de los estudiantes del 2. ${ }^{\circ}$ grado de secundaria de la Institución Educativa 1190 de Chosica, 2018. (Tesis de licenciatura). Universidad Nacional de Educación Enrique Guzmán y Valle. Perú

León, C. (2017). Efectos del programa de estrategias comunicativas para estimular el lenguaje oral en los estudiantes de 5 años de la I.E.I. Los Amiguitos de Lucyana. (Tesis de licenciatura). Universidad Nacional de Educación Enrique Guzmán y Valle. Perú Londoño, D. y Ospina, M. (2018). Comprensión y producción de textos escritos en instituciones de educación superior. Trilogía Ciencia, Tecnología Sociedad, 10(18), pp. $183-202$

Mejía, O., Cortés, O. y Velásquez, A. (2016). Seres y Saberes, Universidad de Tolima, Colombia.

MINEDU (2020). Evaluaciones de logros de aprendizaje. Resultados 2019. [Revisado el 30 de noviembre del 2020] Recuperado desde: https://www.gob.pe/minedu\#publicaciones

Pallares, I. y Rodríguez, G. (2016). El modelo propuesto por Van Dijk y Kintsch y la comprensión lectora de los estudiantes de noveno grado del instituto agrícola de Charta en el municipio de Charta en el departamento de Santander- Colombia 2014. (Tesis de licenciatura), Universidad Privada Norbert Wiener, Perú

Palomino, R. (2017). Comprensión inferencial de textos expositivos de los estudiantes de educación del x ciclo de la especialidad de lengua española y literatura de dos instituciones superiores pedagógicas públicas de lima. (Tesis de maestría), Pontificia Universidad Católica del Perú, Perú

Ripoll, P. (2016). Intervenciones docentes para enseñar a leer ciencias sociales. Resultados de una investigación didáctica desarrollada en un quinto grado de nivel primario. Revista Contextos de Educación, 16 (21), pp 42 - 53. Recuperado desde:

http://www2.hum.unrc.edu.ar/ojs/index.php/contextos/article/view/444/428

Rodríguez, R. (2019). La comprensión lectora: enfoques, niveles, factores y teorías.

(Tesis de bachillerato). Universidad Peruana Unión, Perú

Roncales, F. (2015). Aplicación de talleres literarios con el enfoque comunicativo textual para desarrollar la comprensión lectora de los estudiantes de educación de la ULADECH católica, 2015. Universidad Católica Los Ángeles de Chimbote, Perú 
Rosemberg, C. (2018). El lenguaje y el juego en la educación infantil [Revisado el 30 de noviembre del 2020] Recuperado desde:

https://www.researchgate.net/publication/316845394_El_lenguaje_y_el_juego_en_1

a_educacion_infantil

Sepúlveda, S y Martínez, M. (2018). La importancia de la comprensión lectora: un análisis en alumnado de educación básica en chile. Revista de Educación de la Universidad de Granada, 25, PP. 113-129.

Serrano, G. (2016). El resumen como herramienta de enseñanza-aprendizaje de estrategias para comprender textos narrativos en un colegio particular subvencionado de la V región. (Tesis de licenciatura), Universidad Catolica de Valparaíso, Chile.

sus alumnos en la Educación Primaria. Ocnos. Revista de Estudios sobre lectura, 16 (1), p. $89-106$

Van Dijk, T. (1978). La ciencia del texto. Barcelona, Paidós.

Vela, A. (2017). Macrorreglas de adición para mejorar la comprensión lectora en estudiantes de educación secundaria. (Tesis de Doctorado), Universidad Cesar Vallejo, Perú.

Villalobos, M. y Uceda, S. (2020). Macrorreglas proposicionales y la producción de textos argumentativos en estudiantes de la carrera de educación secundaria, mención Idiomas. Revista CIENCIA Y TECNOLOGÍA, 16(1), pp. 91-99

Wittgenstein, V. (1976). Los cuadernos azul y marrón. Editorial Tecnos, Madrid. 